\title{
LA ACREDITACIÓN EN AMÉRICA LATINA: EL CASO DE ARGENTINA EN LA RIACES Y EN EL MERCOSUR
}

\section{Ernesto Villanueva $(*)$}

SÍNTESIS: Este trabajo detalla dos proyectos de integración educativa regional que funcionan actualmente: el MEXA, en el ámbito del MERCOSUR, y la RIACES, en un contexto que incluye a diversos países iberoamericanos. Por otro lado, se presentan las principales funciones de evaluación y acreditación desarrolladas por la Comisión Nacional de Evaluación y Acreditación Universitaria ( CONEAU) de Argentina, y se explica cómo esta agencia se ha incorporado a los proyectos de integración antes mencionados. La reflexión final apunta a mostrar cómo el desafío que implica la globalización y el progreso de la educación transnacional puede transformarse en una oportunidad para avanzar con proyectos de integración de los sistemas de educación superior de los países de la región.

SÍNTESE: Este trabalho especifica dois projetos de integração educacional regional que funcionam atualmente: O MEXA, no âmbito do MERCOSUL, e RIACES, em um contexto que inclui diversos países ibero-americanos. Por outro lado, apresentam-se as principais funções de avaliação e acreditação desenvolvidas pela Comissão Nacional de Avaliação e Acreditação Universitária (CONEAU) da Argentina, e explica-se como esta agência se incorporou aos projetos de integração antes mencionados. A reflexão final aponta como o desafio que implica a globalização e o progresso da educação transnacional pode transformar-se em uma oportunidade para avançar com projetos de integração dos sistemas de educação superior dos países da região.

(*) Presidente dela Comisión Nacional de Evaluación y Acreditación Universitaria (CONEAU), Argentina. 


\section{LA CALIDAD COMO POLÍTICA PARA LA REGULACIÓN DEL SISTEMA UNIVERSITARIO ARGENTINO}

Desde mediados de la década de los 80 , la evaluación de la calidad de la educación universitaria se ha presentado como uno de los principales temas en la agenda de las reformas educativas en casi todas las regiones del mundo. La evaluación, y más en concreto la acreditación - entendida como una garantía pública de que la carrera o la institución cumplen con un conjunto previamente definido de criterios y de estándares y con los propósitos que ella misma comprometió ante la sociedad-, aparecieron como herramientas adecuadas para regular a través del Estado el sistema de educación universitaria desde la perspectiva de la calidad de los servicios educativos ofrecidos.

En Argentina, la preocupación por la evaluación como instrumento de control de la calidad de la educación quedó reflejada en la Ley de Educación Superior ( LES) n.24.521, sancionada en 1995. En dicha Ley se facultó al Estado para ejercer las funciones de control de la calidad de la educación universitaria, mediante la creación de un organismo dedicado de manera específica a tales tareas: la Comisión Nacional de Evaluación y Acreditación Universitaria, CONEAU, que comenzó sus actividades en agosto de 1996.

La CONEAU es un organismo autónomo que funciona bajo la jurisdicción del Ministerio de Educación. Está integrada por 12 miembros y por diversos equipos técnicos. Los miembros son personalidades de reconocida trayectoria académica y científica, a los que se designa por nombramiento del poder ejecutivo nacional, y que representan al Consejo Interuniversitario Nacional, a la Academia Nacional de la Educación, a ambas cámaras del Congreso Nacional, yal Ministerio de Educación. Sus cargos son de renovación parcial cada cuatro años.

Las funciones de la CONEAU están vinculadas a los procesos de evaluación y de acreditación, y podrían sintetizarse en cuatro tipos de actividades:

a) Realización de evaluaciones externas: Ia LES estipula que las instituciones universitarias deberán ser evaluadas externamente por la CONEAU, como mínimo cada seis años, y que dicha evaluación se llevará a cabo en el marco de los objetivos definidos por cada institución. Estas evaluaciones complementan las autoevaluaciones que efectúan los establecimientos para analizar sus logros y las dificultades con las que se 
encuentran, y para sugerir medidas orientadas al mejoramiento de la calidad. Las evaluaciones externas tienen como principal objetivo asistir a las instituciones en sus propuestas de mejoramiento de la calidad.

En un primer momento, la práctica de la evaluación fue considerada por muchas instituciones como un mecanismo encubierto para conocer la situación de las universidades, y, a partir de esos datos, para establecer una especie de ranking de calidad, que, a su vez, implicaría penalizaciones o condicionaría la distribución de fondos. Sin embargo, poco a poco las instituciones fueron adoptando la práctica de la evaluación externa, como una instancia de diagnóstico a partir de la cual se pudiera reflexionar sobre su propia situación e introducir cambios que condujeran a su mejora. La CONEAU tiene mandato legal para realizar estas evaluaciones, y, además, brinda el soporte técnico y profesional de las mismas.

A fines de 2003, 37 instituciones universitarias ya habían sido evaluadas, y los informes que se elaboraron con este propósito pueden consultarse en la página web de la CONEAU ${ }^{1}$. Por otra parte, a mediados de 2004 otras cinco instituciones se encuentran en proceso de evaluación, y 20 más han firmado acuerdos para iniciar sus respectivos procesos de evaluación.

b) Recomendación deacreditación de proyectos institucionales: a través de minuciosos procesos de evaluación, la CONEAU elabora informes sobre la consistencia y la viabilidad de proyectos institucionales de universidades públicas y privadas. Dichos informes son remitidos al Ministerio de Educación, instancia que aprueba o no el funcionamiento de los proyectos en cuestión, les otorga reconocimiento provisional y luego otro definitivo. Si bien la CONEAU actúa como asesora del Ministerio, sus informes son vinculantes, es decir, que para que una nueva universidad funcione legalmente tiene que contar con el informe favorable de la Comisión.

Dado el importante aumento registrado en la cantidad de instituciones universitarias, sobre todo de las privadas, durante los primeros años de la década de los 90 , la puesta en marcha de los procesos de acreditación institucional han sido de suma importancia. Entre 1990 y 1995 el Poder Ejecutivo había autorizado el funcionamiento de 23

\footnotetext{
${ }^{1}$ http://www. coneau. gov. ar.
} 
nuevas instituciones, casi la misma cantidad que la creada en los 30 años anteriores. Una vez que laCONEAU inició sus actividades en 1996, y hasta finales de 2003, sólo nueve establecimietos que se habían creado durante ese período consiguieron autorización para llevar a cabo sus labores. Este fue el resultado de la evaluación de los 72 proyectos presentados: 29 obtuvieron dictamen negativo, 34 fueron retirados antes de la resolución - vistos los informes preliminares del expediente-, y sólo 9 alcanzaron el reconocimiento provisional. A comienzos de 2004 la CONEAU remitió cuatro dictámenes más sobre creación de instituciones al Ministerio, e inició la evaluación de otros tantos proyectos. También en los últimos años se han expedido resoluciones sobre cinco proyectos de creación de universidades nacionales: cuatro consiguieron dictámenes favorables y uno fue rechazado.

c) Acreditación de carreras consideradas de interés público: el artículo 43 de la LES establece que aquellas carreras cuyo ejercicio profesional pueda poner en riesgo la salud, la seguridad, los derechos, los bienes o la formación de los habitantes, deberán ser acreditadas por la CONEAU. Esta actividad se realiza a través de una detallada evaluación hecha a partir de estándares consensuados entre el Ministerio de Educación y el Consejo de Universidades, y la Comisión se pronuncia autorizando o no el funcionamiento de estas carreras.

La obligatoriedad de la acreditación para las carreras reguladas por el Estado funciona de manera retroactiva, dado que las que ya están funcionando también deben presentarse a la CONEAU para ser evaluadas y acreditadas. Las carreras de Medicina y de Ingeniería de todas las universidades del país han pasado ya por los procesos de acreditación, mientras la de Agronomía está actualmente siendo analizada, y se prevé que otras profesiones queden sujetas igualmente a esta disposición (Farmacia, Bioquímica, Veterinaria, Arquitectura, Odontología, entre otras). Acerca del proceso de acreditación de las carreras de Agronomía y de Ingeniería nos ocuparemos más adelante, ya que dicho proceso se realiza en el marco de instancias supranacionales como el MEXA (Mecanismo Experimental de Acreditación del MERCOSUR).

d) Acreditación obligatoria de todas las carreras de postgrado: el crecimiento de la oferta de postgrados constituye una de las transformaciones más notables ocurridas en la década de los 90 en el sistema de educación superior: entre 1994 y 1999 los postgrados se incrementaron en un $168 \%$. Cierto es que Argentina tenía un retraso considerable en esta área, con lo cual los índices de crecimiento son más abultados, pero, 
de todos modos, queda claro que el ámbito de los postgrados se está transformando con rapidez. A partir de la promulgación de la LES, se contó con un instrumento legal para regular ese desmesurado crecimiento.

En realidad, los procesos de acreditación de los postgrados se iniciaron en 1994, año en el que se creó la Comisión de Acreditación de Postgrados (CAP). Este organismo estuvo encargado de comenzar los procesos de acreditación de maestrías y de doctorados presentados de manera voluntaria. Su trabajo dio como resultado la acreditación de 99 maestrías y de 77 doctorados, más o menos un $59 \%$ de las carreras presentadas. Con la puesta en vigor de la LES y la correspondiente creación de la CONEAU, la CAP se disolvió y sus funciones fueron traspasadas a aquella.

Es notable el papel jugado por la CONEAU en estos procesos, dado que casi todo el universo de las carreras de postgrado, al rededor de 2.150, se han presentado para ser acreditadas por la Comisión: 306 doctorados (14\%); 798 maestrías (37\%); y 1.047 especializaciones (49\%). En este sentido, la CONEAU ha logrado legitimar su función de acreditadora, yha asegurado bases mínimas de calidad para los postgrados ofrecidos en el país.

e) Recomendación de reconocimiento de entidades privadas de evaluación y de acreditación: la LES faculta la posibilidad de que se constituyan entidades privadas de evaluación y de acreditación, pero, para poder funcionar, deberán contar con un previo informe favorable de la CONEAU. Hasta el momento se ha aprobado un proyecto de creación de agencia privada, y se comienza a analizar otro.

\section{LOS DESAFÍOS DE LA AGENCIA ACREDITADORA EN EL CONTEXTO DE LA GLOBALIZACIÓN}

Desde su puesta en marcha en 1996, laCONEAU ha desarrollado una intensa labor, producto de la cual se han evaluado y acreditado carreras e instituciones, tal y como estipula la Ley. No obstante este fructífero desarrollo, la CONEAU ha tenido que encarar durante estos años desafíos que en algunos casos han obstaculizado su tarea, pero que en otros se han convertido en importantes estímulos para avanzar en su consolidación acreditadora. 
EI primer gran desafío al que tuvo que hacer frente la CONEAU fue el de construir y legitimar su lugar como agencia evaluadora y acreditadora, en un medio universitario que al principio no recibió con beneplácito su creación. Cuando la CONEAU comenzó sus tareas, muchos actores universitarios manifestaron su oposición a la Comisión, suponiendo que la evaluación y la acreditación pondrían en cuestión el principio de la autonomía universitaria, al otorgar al Estado instrumentos de control sobre el sistema universitario. Por fortuna, la sistemática y responsable tarea realizada por la CONEAU ha demostrado que su misión no es incompatible con el principio de la autonomía universitaria, y que, más aún, es superadora del control formal y burocrático que realizaba el Estado. Las evaluaciones se han convertido en una herramienta para las propias instituciones, en un diagnóstico a partir del cual se pueden realizar ajustes y transformaciones. La legitimidad de la Comisión y de la práctica de la evaluación también ha estimulado las propias estructuras de las universidades, la creación de áreas dedicadas específicamente a la evaluación, y la autoevaluación institucional y de las carreras.

Otro gran desafío para la Comisión ha sido -y lo sigue siendodiseñar y aplicar herramientas técnicas que permitan llevar a cabo una labor productiva y justa: productiva, para que la evaluación y la acreditación no sean sólo trámites formales y burocráticos, sino que respondan al espíritu con el que fueron propuestos en la Ley, esto es, actuar como instancias que faciliten el mejoramiento de la educación universitaria y aseguren la calidad de lo que se ofrece. Más difícil aún ha sido lograr que los instrumentos utilizados para los procesos de evaluación y de acreditación conduzcan a resultados justos y objetivos, es decir, que reduzcan las arbitrariedades y las subjetividades del caso. En este sentido, el desafío técnico se renueva cada vez que la CONEAU se enfrenta a una nueva tarea, y en parte sólo la experiencia acumulada, los errores y los aciertos, sirven para superar los obstáculos.

Ahora bien, la agencia acreditadora igualmente se enfrenta hoy a importantes retos que provienen del plano externo, y que tienen que ver con los procesos de globalización y de internacionalización de la educación.

Los tradicionales sistemas de educación superior, organizados a partir de instituciones universitarias o terciarias con modalidades presenciales, están siendo desafiados por el desarrollo de la educación transnacional en todas sus formas, por las aplicaciones tecnológicas al área educativa, y por diversos proyectos políticos que intentan disolver las barreras que impiden el intercambio de servicios educativos entre 
distintos países, situación que muchos ya empiezan a llamar el «mercado de la educación».

No obstante los riesgos de ese impacto -sobre todo en los sistemas de educación latinoamericanos, que son fácilmente convertidos en receptores de los servicios educativos exportados por los países centrales-, también es verdad que la globalización ha revitalizado y encuadrado de manera más sólida los proyectos de integración regional. En tales proyectos, la educación superior ha comenzado a ser uno de los ejes concretos para trabajar políticas de integración: la idea de una región sin fronteras ha estimulado la discusión sobre los problemas comunes de los sistemas de educación, y sobre las posibilidades de concretar proyectos de integración educativa a nivel regional.

Si bien en Argentina no se cuenta con una legislación demasiado específica de cara al desafío que presenta la educación transnacional, sí ha podido incorporarse a los proyectos regionales, y, en ese sentido, convertir el reto de la gl obalización en una oportunidad. LaCONEAU, como agencia acreditadora del Estado, participa activamente en dos proyectos sobre evaluación y acreditación regional: laRIACES y el MEXA².

\section{EL MERCOSUR Y LA INTEGRACIÓN PARA LA ACREDITACIÓN DE CARRERAS: EL MEXA}

EI MERCOSUR ha instituido un programa vinculado a las cuestiones educativas: el MERCOSUR Educativo. Dentro de ese programa, los países de la subregión, junto con Bolivia y Chile, están trabajando acerca de espacios de discusión sobre temas de acreditación y de evaluación. La CONEAU participa, con voz y voto, en las reuniones de las Agencias Nacionales de Acreditación, en las del Comité Regional Coordinador de Educación Superior, y en las del Grupo de Trabajo de Expertos en Evaluación y Acreditación Universitaria. En los diversos encuentros realizados, los principales objetivos han sido siempre poder armonizar criterios y diseñar procesos de evaluación y acreditación universitaria

2 La CONEAU también participa en otros espacios internacionales: es miembro pleno de la Red Internacional de Agencias de Aseguramiento de la Calidad de la Educación Superior [International Network of Quality Assurance of Higher Education (INQAAHE)]; participa en las discusiones de la Alianza Global para la Educación Transnacional [Global Alliance for Transnational Education (GATE)], y mantiene vínculos con instituciones acreditadoras de otros países. 
conjuntos entre los países que integran el MERCOSUR, con las finalidades de contribuir al proceso de integración regional, de promover la mejora permanente de los sistemas educativos nacionales, y de facilitar la libre circulación de factores (estudiantes, profesores y profesionales).

En ese marco, los ministros de educación de los países del MERCOSUR, junto con los de Bolivia y Chile, firmaron en 1998 el «Memorando de Entendimiento sobre la implementación de un mecanismo experimental de acreditación de carreras para el reconocimiento de títulos de grado universitario en el MERCOSUR», que estableció el diseño de un Mecanismo Experimental para el desarrollo de las actividades relacionadas con la Evaluación y la Acreditación Universitaria (MEXA). En los años siguientes se trabajó intensamente en la elaboración conjunta de instrumentos adecuados para la implementación, y en cuestiones referentes a la articulación de ese proceso regional con los procesos nacionales y con la capacitación de recursos humanos. La tarea dio comienzo con la acreditación de las carreras de Agronomía, proceso que ya está en su etapa final; en el 2004 se está trabajando en la convocatoria para las carreras de Ingeniería y de Medicina, como se explicará más adelante

Por acreditación se entiende el proceso mediante el cual se otorga validez pública, en tanto calidad académica y de acuerdo con las leyes nacionales, a los títulos universitarios. La acreditación es periódica, de acuerdo con parámetros de calidad previamente definidos para el MERCOSUR, y está recogida en el documento «Dimensiones, Componentes, Criterios e Indicadores». Todos los estándares utilizados para la evaluación de las carreras seleccionadas (Agronomía, y ahora Ingeniería y Medicina) fueron definidos por las comisiones consultivas, integradas por expertos de los distintos países y aprobados al comenzar este mecanismo de acreditación. Las Agencias Nacionales de Acreditación, como la CONEAU, conducen este procedimiento en sus respectivos países.

La adhesión al MEXA es voluntaria, y convoca a aquellas carreras que cuenten con reconocimiento oficial en el país yque tengan egresados. En el caso de Argentina se presentaron 18 carreras, de las cuales fueron seleccionadas cinco, en la forma como establece el Mecanismo, adoptándose el criterio de antigüedad para dicha selección ${ }^{3}$.

\footnotetext{
${ }^{3}$ En Argentina, las carreras seleccionadas correspondieron a las siguientes instituciones: U niversidad de Buenos Aires (Facultad de Agronomía); Universidad Nacional de Cuyo (Facultad de Ciencias Agrarias); Universidad Nacional de La Plata (Facultad de Ciencias Agrarias y Forestales); U niversidad Nacional de Tucumán (Facultad de Agronomía y Zootecnia); Universidad Nacional del Nordeste (Facultad de Ciencias Agrarias).
} 
El proceso de acreditación incluye la presentación de un informe institucional y de una autoevaluación, realizadas en un período de hasta cuatro meses, siguiendo las pautas establecidas en los instrumentos preparados por la CONEAU, y de acuerdo con lo aprobado por el MEXA. U na segunda etapa es la selección, el entrenamiento y la actuación de comités de pares, que analizan esos informes y otros datos pertinentes, que realizan la visita a la sede de la carrera y que elaboran un dictamen respecto de su calidad. Los pares deben tener al menos diez años de ejercicio profesional, ser reconocidos como expertos en su área, y estar recomendados por un representante de una institución de prestigio en el ámbito académico o de la profesión. Cada país propone aproximadamente 20 pares. Por otro lado, cada comité de pares debe incluir al menos dos representantes de diferentes Estados-parte 0 asociados al MERCOSUR distintos a los del país al que pertenece la carrera que se va a evaluar.

Una vez que los pares elaboran los dictámenes, la agencia de acreditación del país en el que se ofrece la carrera evaluada otorga o deniega dicha acreditación, siguiendo los procedimientos establecidos por la propia agencia, y de acuerdo con los criterios de calidad establecidos por el MEXA. La decisión de la agencia se comunica a la reunión de Ministros de Educación del MERCOSUR, y a los de Bolivia y Chile. En el caso de que la carrera no cumpla satisfactoriamente con algunos de los criterios esenciales, puede resolverse la postergación del dictamen, y, de no presentar un plan de mejoramiento aceptable, no se la acredita. La institución no podrá presentar otra vez la misma carrera a la acreditación antes de transcurridos dos años. La información y la publicidad de los dictámenes y de las resoluciones deberán referirse sólo a las carreras acreditadas.

Salvo Brasil y U ruguay, las respectivas agencias de acreditación ya han entregado los informes preliminares de los pares de las carreras evaluadas (marzo de 2004), las cuales contestaron a la vista de esos informes. Un mes después, en abril, los pares comenzaron a trabajar en el informe final, que será presentado en la próxima Reunión de Ministros de Educación.

Según el calendario de trabajo acordado, las agencias acreditadoras se encuentran laborando en el taller de pares para las carreras de Ingeniería, cuya fase final tendrá lugar en Ciudad del Este (Paraguay). Cada país efectúa dicha convocatoria a las carreras de Ingeniería para el MEXA, siendo seis por país en tres especialidades. 
Argentina ha seleccionado para esta primera convocatoria las especialidades de Ingeniería Electrónica, Ingeniería Química e Ingeniería Industrial. Mientras tanto, se ha planeado para el segundo semestre de este año la convocatoria para las carreras de Medicina, con el fin de iniciar los respectivos procesos.

EI MEXA es una de las primeras medidas concretas para dar mayor fluidez al contacto académico entre los países del MERCOSUR, más Bolivia y Chile, medidas que son de vital importancia para maximizar la calidad educativa en estos países, y para adecuar los criterios requeridos por la actual sociedad de la información. EI MEXA permite, además, conocer las realidades educativas de los países de la región, integrando y compartiendo problemas y soluciones.

De momento, se trata de trabajar en un plano que permita establecer estándares y criterios comunes para la evaluación y la acreditación. Por su parte, corresponderá a las instancias políticas producir los acuerdos necesarios que faciliten el reconocimiento de títulos y de credenciales.

\section{HACIA LA INTEGRACIÓN IBEROAMERICANA: LA RED IBEROAMERICANA PARA LAACREDITACIÓN DE LA CALIDAD DE LA EDUCACIÓN SUPERIOR}

En un marco regional mucho más amplio, en mayo de 2003 se constituyó la RIACES (Red Iberoamericana para la Acreditación de la Calidad de la Educación Superior). Ya en enero de 2002, los países de América Latina, el Caribe y España, comenzaron a trabajar en un espacio de colaboración y de intercambio, con los objetivos de profundizar en el conocimiento mutuo de sus sistemas universitarios, de identificar problemáticas comunes sobre los procesos de acreditación, y de promover proyectos de cooperación y de transferencia que faciliten la puesta en marcha de procesos de evaluación y de acreditación regional. En abril de 2003, en las dependencias de la CONEAU en Buenos Aires, la Red quedó formalmente constituida mediante la elección de autoridades y la aprobación del Estatuto correspondiente.

Actualmente la Red está integrada por las agencias de acreditación de Argentina, Bolivia, Brasil, Chile, Colombia, Costa Rica, República Dominicana, Ecuador, El Salvador, España, México, Nicaragua, Panamá, Paraguay, Perú, U ruguay y Venezuela; también participan algunos 
organismos internacionales como la Organización de Estados Iberoam ericanos (OEI).

El principal objetivo de la RIACES es crear un espacio de conocimiento recíproco, de cooperación técnica y de intercambio humano e intelectual entre los sistemas universitarios, potenciando los fines similares que presidieron la creación de las agencias y de las unidades de evaluación y de acreditación en cada uno de los países. Por otro lado, la RIACES aspira a convertirse en una herramienta de integración de los países iberoamericanos, que facilite el diseño y la puesta en práctica de respuestas conjuntas y coordinadas frente al desafío que surge del proceso de globalización de la educación superior.

Para cumplir con dichas aspiraciones la RIACES ha creado un portal en Internet, http://www.riaces.org, el cual, además de facilitar la comunicación y la interacción entre los países miembros, permite generar espacios de reflexión, de debate y de información para todos los interesados en el tema de la educación superior.

En febrero de 2004 se realizó la primera Asamblea General de la Red en La Habana, dentro del Seminario Internacional Universidad 2004, organizado por el Ministerio de Educación Superior de Cuba. Dicha reunión contó con la participación de representantes de la mayoría de los países miembros de la Red, quienes discutieron sobre el trabajo y sobre el funcionamiento de la misma. Antes de este encuentro, la Red estaba organizada en cuatro comisiones: Estudios Comparados entre los países de la región; Gestión y mantenimiento del portal; Organización de Talleres de Formación para Evaluadores, y Diseño de un software para la autoevaluación. En el marco de la asamblea se crearon otras tres comisiones: Glosario de términos de evaluación y de acreditación; Buenas prácticas en evaluación y acreditación; y Estudio comparativo de títulos. Las comisiones continúan trabajando, y la información sobre sus avances puede consultarse en el portal.

Como se ve, el trabajo de laRIACES apunta a desarrollar espacios de intercambio, de conocimiento y de labor cooperativa. Si bien no se ha avanzado en el diseño de programas de acreditación conjunta, en el portal hay sitios de discusión sobre estos asuntos, y se han promovido actividades diversas en las cuales los temas principales fueron la evaluación y la acreditación. 


\section{A MANERA DE SÍNTESIS: TRANSFORMAR LOS DESAFÍOS EN OPORTUNIDADES}

La globalización no se ha expandido pensando sólo en las necesidades de los países latinoamericanos. Desde esa perspectiva regional, es posible reconocer una primera etapa, caracterizada por una especie de internacionalización de partes del Estado mediante las privatizaciones, de un avance de la concentración bancaria en manos de capitales extranjeros, de la mayor injerencia de los organismos financieros internacionales en el diseño de las políticas económicas locales, de la desindustrialización, de un librecambio exagerado, y de las dificultades crecientes de los Estados para regular tales fenómenos.

A esta primera etapa de reformas le ha seguido una segunda «ola», centrada ya no tanto en las actividades productivas, extractivas, de transporte y de comunicaciones, sino en servicios que tradicionalmente se han considerado bienes «no transaccionales», no aptos para ser intercambiados en el comercio internacional. Y, entre estos bienes, la educación superior ha comenzado a aparecer como centro de las disputas entre aquellos que pregonan la necesidad de desregular el mercado internacional de la educación, y quienes siguen sosteniendo que la educación nunca puede ser considerada como una mercancía.

Como ya se dijo, los países latinoamericanos se encuentran en una situación de relativa debilidad frente al desarrollo de la educación transnacional. Por un lado, porque en muchos de ellos los sistemas de educación superiorno son demasiado sólidos, y porquela cuestión de la educación transnacional se ha incorporado a sus agendas de problemas educativos de forma reciente. Por otro lado, porque los informes de los organismos mundiales acerca de los índices de pobreza, de desigualdad y de perspectivas de desarrollo no son muy alentadores, con lo cual los contextos social, político y económico tampoco estarían colaborando en la mejora de los sistemas de educación universitaria.

En este sentido, ni el MEXA ni la RIACES son una panacea, pero tampoco deben considerarse como reacciones espontáneas de países amenazados. Por el contrario, constituyen proyectos educativos regionales que están convirtiendo los desafíos de la gl obalización en oportunidades para la integración, borrando las fronteras donde éstas se muestran como un obstáculo para el mutuo conocimiento, el intercambio y la integración, pero respetándolas cuando dichas fronteras resguardan identidades y formas culturales particulares. 
En el caso del MEXA, la perspectiva sobre la que se está avanzando es concreta, no sólo declarativa; prueba de ello son los resultados de la acreditación de las carreras de Agronomía, la convocatoria para las de Ingeniería, y los documentos que preparan la convocatoria para las carreras de Medicina. El tiempo dirá en qué medida estas carreras han podido mejorar, y en qué sentido cada sistema nacional de educación superior se ha visto beneficiado por los intercambios y por la integración. De momento, el MEXA se encuentra en una etapa de mucho trabajo y experimentación.

Por otro lado, Ios avances del MEXA también han estimulado el desarrollo de otras líneas de trabajo en el marco del MERCOSUR Educativo. Recientemente se han aprobado diversos documentos que facilitan la residencia legal de estudiantes, de docentes e investigadores y de sus respectivas familias en otro de los países miembros durante el período que requieran sus estudios o sus trabajos. Estos acuerdos suscritos también prevén el reconocimiento de tramos de carreras a partir de la fijación de determinadas cargas horarias.

EI caso de la RIACES abre otro tipo de oportunidades, dado que aún se presenta básicamente como un espacio para el intercambio de conocimientos y de experiencias. De todos modos, una dimensión regional más amplia y una mayor cantidad de instituciones y de expertos involucrados, hace más compleja y enriquecedora la tarea que se ha propuesto. Por otro lado, la formación de la RIACES es relativamente nueva, con lo cual es de esperar que en un par de años también avance sobre proyectos y programas de integración más concretos.

Defender la identidad cultural y la equidad como variables inseparables de la calidad de los productos y de los servicios educativos que se ofrecen, es, tal vez, el mayor desafío para la comunidad iberoamericana. Las agencias evaluadoras y acreditadoras deben seguir aunando esfuerzos para analizar, para reflexionar y para aplicar criterios en pos de tal objetivo.

\section{BIBLIOGRAFÍA}

BOLETín ACREDITAR núms. 1, 2, 3, 4 (2003): http://www.coneau.gov.ar/boletin, Buenos Aires, CONEAU. 


\section{E. VILLANUEVA}

CONTRIBUCIONES PARA UN ANÁlisis del ImPACTO DEL Sistema de EVALUACIÓN Y ACREDITACIÓN (2002): Documento de Trabajo, Buenos Aires, CONEAU.

CORAGGIO, J. L. y VISPO, A. (coords.) (2001): Contribuciones al estudio del sistema universitario argentino, Buenos Aires, Miño y Dávila, CIN.

FERNÁNDEZ LAMARRA, N. (2003): La educación superior argentina en debate. Situación, problemas y perspectivas, Buenos Aires, Eudeba-IESALC/UNESCO.

Garcíade FAnelli, A. M. (1999): La educación transnacional: la experiencia extranjera y lecciones para el diseño de una política de regulación en la Argentina, Buenos Aires, CONEAU.

LAS H ERAS, J . yRosselot, E. (comps.) (2004): Calidad en M edicina. Criterios para la acreditación de programas de facultades de Ciencias de la Salud, Universidad de Chile, CIDAFAM.OSDE.

Pugliese, J. C. (ed.) (2003): Políticas de Estado para la Universidad Argentina. Balance de una gestión en el nuevo contexto nacional e internacional, Buenos Aires, Ministerio de Educación, Ciencia y Tecnología, Secretaría de Políticas Universitarias.

SÁNCHEZ M. E. (ed.) (1999): La educación superior en la Argentina. Transformaciones, debates, desafíos, Buenos Aires, Ministerio de Educación, Ciencia yTecnología, Secretaría de Políticas Universitarias.

REVISTALA UNIVERSIDAD (n. 18) (1999): «Universidad y globalización», Buenos Aires, Ministerio de Educación, Ciencia y Tecnología, Secretaría de Políticas Universitarias.

\section{SITIOS DE INTERÉS}

- http://www.coneau.gov.ar.

- http://www.me.gov.ar/mercosur.

- http://www.mercosul.mec.gov.br/default.asp.

- http://www.inqaahe.nl.

- http://www.riaces.org. 


\title{
Contactar
}

Revista lberoamericana de Educación

\author{
Principal OEI
}

